A N N A L E S Annales de Bretagne et des Pays de l'Ouest

\title{
Mortalité et hygiène dans la ville de León au cours de l'époque moderne
}

María José Pérez Álvarez

\section{(2) OpenEdition}

\section{Journals}

Édition électronique

URL : http://journals.openedition.org/abpo/106

DOI : 10.4000/abpo.106

ISBN : 978-2-7535-1514-7

ISSN : 2108-6443

Éditeur

Presses universitaires de Rennes

Édition imprimée

Date de publication : 30 juin 2009

Pagination : $55-78$

ISBN : 978-2-7535-0941-2

ISSN : 0399-0826

\section{Référence électronique}

María José Pérez Álvarez, " Mortalité et hygiène dans la ville de León au cours de l'époque moderne »,

Annales de Bretagne et des Pays de l'Ouest [En ligne], 116-2 | 2009, mis en ligne le 30 juin 2011, consulté le 30 avril 2019. URL : http://journals.openedition.org/abpo/106 ; DOI : 10.4000/abpo.106 


\title{
Mortalité et hygiène dans la ville de León au cours de l'époque moderne
}

\author{
María José PÉREZ ÁlVAREZ \\ Professeur titulaire d'histoire moderne, \\ Université de Léon
}

L'objectif du présent travail est d'analyser les crises de mortalité qui ont eu lieu dans la ville de Léon durant l'époque moderne et les facteurs qui, directement ou indirectement, les ont provoquées. Un deuxième aspect de l'étude s'intéressera aux interventions des pouvoirs publics pour enrayer le phénomène de la mortalité.

\section{Cadre spatial et chronologique}

La ville de Léon fait partie de l'actuelle Communauté autonome (Comunidad Autónoma) de Castille et León, mais son évolution historique n'a pas suivi la même évolution démographique et économique que celle du réseau urbain castillan, beaucoup plus connu par l'historiographie (Valladolid, Burgos, Ségovie...). Durant la période d'étude, sa population a oscillé entre 3846 habitants en 1591 et 6051 en 1787, sans atteindre le seuil des 10000 , chiffre donné par certains auteurs pour mesurer le degré d'urbanisation, avant $1860^{1}$. La configuration topographique de la ville doit beaucoup à ses origines romaines ainsi qu'à l'évolution postérieure tout au long du Moyen Âge. L'enceinte romaine a été franchie au Moyen Âge du fait de l'expansion démographique de la cité2. Par ailleurs, des fondations religieuses se sont installées hors les murs, formant ainsi les faubourgs. Ces espaces péri-urbains avaient un caractère nettement agricole, avec des vergers, des prés et des linières. Ils regroupaient aux alentours de $20 \%$ des habitants de Léon entre la fin du Xvi et le milieu du XVIII siècle, période où le nombre d'habitants a augmenté d'un peu plus de $40 \%{ }^{3}$. Bien que les

1. Voir De VRIES, Jan, La Urbanización de Europa, 1500-1800, Barcelone, Crítica, 1987.

2. Álvarez Álvarez, Cesar, La Ciudad de León en la Baja Edad Media, Léon, Sociedad Anónima Hullera Vasco-Leonesa, 1992, p. 41-50.

3. RuBio PÉREz, Laureano Manuel, León 1751. Según las Respuestas Generales del Catastro de Ensenada, Madrid, Tabapress, 1992, p. 52. 
paroisses extra-muros aient connu la plus forte croissance (66\%), celles de l'intérieur ont aussi profité de cet essor démographique en augmentant de plus d'un tiers. Ce processus a eu pour conséquence un entassement de la population sans précédent dans un périmètre urbain qui était pratiquement le même que deux siècles auparavant.

Carte 1 - Localisation géographique de la province de León

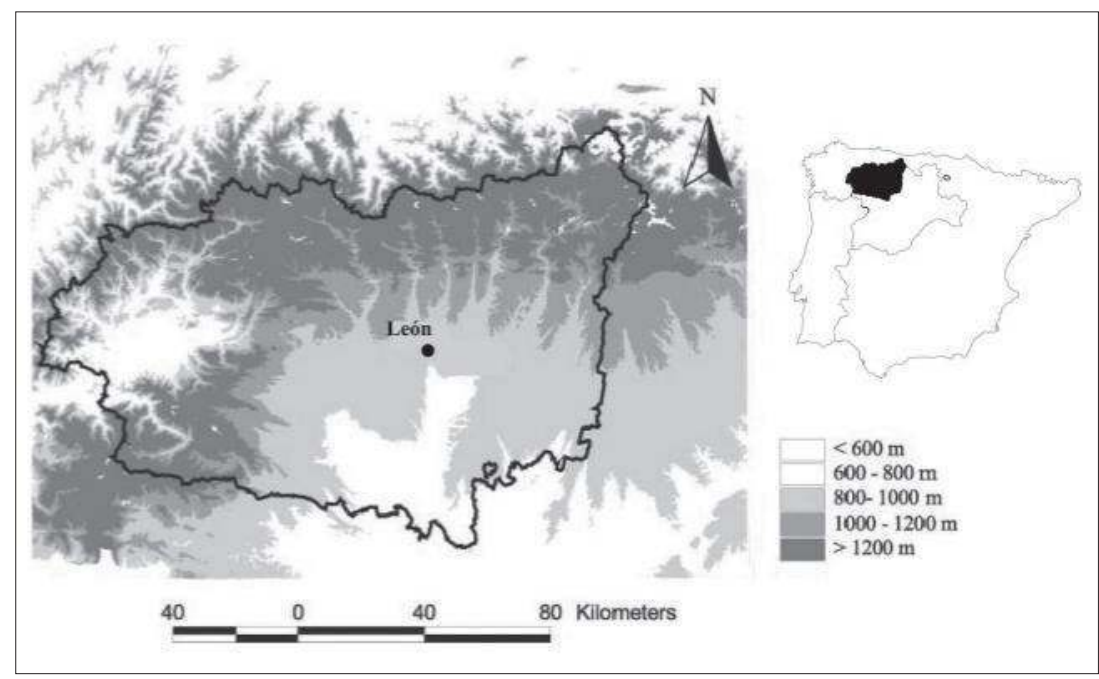

Léon resta longtemps une ville à caractère rural où le poids des secteurs secondaire et tertiaire était peu important. Au milieu du XVIII ${ }^{\mathrm{e}}$ siècle, la société de Léon était fortement polarisée et hiérarchisée. La faible présence de la Hidalguía 4 , 1,9 \% des habitants en 1752, n'a pas empêché celle-ci, unie au clergé et à un petit groupe d'administratifs, de contrôler 20 \% des moyens de production, en particulier la propriété de la terre autour de la ville, les troupeaux et les moulins qui constituaient le principal potentiel de richesse. Une partie de ce groupe, la noblesse titrée et la Hidalguía, possédait le pouvoir politique dans la ville. Certains de ses membres ont eu accès au conseil municipal car ils étaient de proches parents de la vieille noblesse de Léon, celle qui, au Xvie siècle, était allée à la Cour à la recherche d'une influence qu'elle ne pouvait pas obtenir dans une capitale de province. Ces nouveaux dirigeants, conjointement avec d'autres familles de la bourgeoisie commerciale, bénéficièrent de la crise du XVII ${ }^{\mathrm{e}}$ siècle, vu que le déficit du Trésor royal (Hacienda Real) leur permit de se maintenir dans leurs postes comme gouvernants permanents. Contrôlant les relations de dépendance sociale grâce à ces postes, ils se retrouvaient à la tête des

4. N.T. : Hidalguía est la noblesse qui vient aux individus par leur lignage, était hidalgo qui prouvait la noblesse de son père et de son grand-père. 
moyens de production, grâce à quoi ils ont pu exercer toute leur influence, bloquer toute innovation qui pouvait leur paraître défavorable, comme le prouve l'échec du projet de la Real Fábrica de Lienzos (fabrique royale de toiles) au milieu du XvIII siècle.

Pour analyser les étapes et les effets des crises de mortalité sur la ville de Léon pendant l'époque moderne, qui, nous le verrons, ont une chronologie assez similaire à celle qui a été observée dans d'autres points de la péninsule, nous disposons de deux sources complémentaires. En premier lieu, les registres paroissiaux dont la consultation est obligatoire dans ce type de travaux pour la période qui nous occupe. Nous avons dépouillé, avec des résultats divers, les fonds des principales paroisses de la ville : Santa María del Mercado, San Martín, San Juan de Regla, Santa Ana, Palat de Rey et Santa Marina la Real. Les paroissiens formaient au Xvi ${ }^{\mathrm{e}}$ siècle 73,6 \% des familles qui habitaient dans la ville. Au XvIII siècle, ils représentaient $68 \%$ des familles de l'échantillon dont nous disposons ${ }^{5}$. Les registres de mortalité d'adultes ne sont continus qu'à partir de la deuxième décennie du xvII siècle, ce qui nous oblige à analyser les comportements démographiques du Xvie siècle exclusivement à travers les actes de baptême dont nous disposons de manière continue depuis 1523 , mais pas pour toutes les paroisses. Quant à la mortalité infantile, il est préférable de renoncer à son analyse jusqu'au milieu du XVIII ${ }^{e}$ siècle en raison des lacunes des sources.

En complément des registres paroissiaux, nous utiliserons les actes et les arrêtés municipaux ainsi que les minutes notariales. Ces actes permettent de compléter et de nuancer les résultats obtenus à partir des registres paroissiaux. Les données qualitatives quy y figurent nous ont permis de mieux connaître, entre autres aspects, la nature des crises, la réponse et les mesures de prévention prises par le pouvoir politique local face à de tels événements ou son intervention en matière de santé préventive.

\section{Les crises de mortalité dans la ville de León durant l'époque moderne}

Le $\mathrm{xvI}^{\mathrm{e}}$ siècle s'est caractérisé en Europe par une forte croissance démographique, qui a affecté également la ville de Léon et sa province ${ }^{6}$. Cependant, ces perspectives favorables se sont évanouies pendant les dernières années du siècle. L'évolution des naissances établie à partir des séries baptismales des paroisses ${ }^{7}$ de San Martín, Santa Marina del Rey, San

5. Les deux pourcentages calculés à partir des recensements de 1591 et 1752 ont été publiés par MARTín GALINDO, José Luís, La ciudad de León en el siglo XVIII, Léon, Instituto Leonés de Cultura, 1957, p. 44.

6. Voir PÉREZ GARCíA, José Manuel, « Demografía leonesa en el Antiguo Régimen (1500 1850) ", dans Rubio PÉREz, Laureano (coord.), Historia de León, Vol. III, León, Université de Léon, 1999, p. 188-211, p. 192.

7. Le graphique est élaboré à partir de 1523 et jusqu'à 1621, année à partir de laquelle on dispose de données dignes de foi sur la mortalité dans la ville de Léon. 
Juan de Regla, el Mercado et Palat de Rey, nous permettra d'observer les différents cycles démographiques que la ville a traversés durant un siècle.

Malheureusement, la date tardive de tenue des registres nous empêche de connaître les conséquences que la poussée de peste de 1517-1518 a eues sur la ville ${ }^{8}$. À partir de 1523, et jusqu'à 1619 , nous pouvons diviser l'évolution démographique en trois phases (graphique $\mathrm{n}^{\circ} 1$ ). La première, qui s'étend de 1523 jusque au-delà du milieu du Xvi ${ }^{e}$ siècle, s'est caractérisée par un accroissement. Toutefois, il s'agit d'une croissance très lente, lenteur due probablement à l'incidence d'épisodes pesteux, comme la poussée de 1530 attestée par plusieurs documents ${ }^{9}$. Peut-être ne faut-il pas surestimer celle-ci vu que les indices de baptêmes de cette année sont similaires à ceux des années antérieures et postérieures. Au milieu du Xvie siècle s'ouvre une deuxième phase. À partir de ce moment, la croissance de la population a été telle que nous pouvons affirmer que nous sommes devant la plus grande phase d'expansion de ce siècle. Il semble que les épisodes critiques de 1568-1569 ${ }^{10}$, à propos desquels le conseil municipal de Léon était parfaitement informé, n'ont pas laissé de trop lourdes séquelles, bien que cela ait été ponctuellement le cas en milieu rural ${ }^{11}$. La réalité de cette croissance de la population est attestée par les recensements de 1561 et de $1575^{12}$, avec une croissance annuelle moyenne de 1,98\%. Enfin, la troisième phase a débuté à la fin des années 1580 . C'est à ce moment-là que la tendance démographique commence à s'inverser, bien que le renversement dû à des facteurs exogènes ne se soit fait nettement sentir qu'au cours la décennie suivante. La cause en était une longue période de mauvaises récoltes ${ }^{13}$ qui

8. Cubillo de La Puente, Roberto, " Higiene y sanidad ", dans Rubio Pérez, Laureano M. (coord.), Historia de León, Vol. III. León, Université de León, 1999, p. 444-449, p. 445. Cet épisode de peste coïncide avec celui qui a eu lieu à la même époque à Valladolid.

9. Dans d'autres territoires de l'actuelle Communauté autonome de Castille-Leon, la présence de la peste est attestée à la même date. CARRERAS PANCHÓN, Antonio, La Peste y los médicos en la España del Renacimiento, Université, Instituto de Historia de la Medicina Española, Salamanque, 1976.

10. Cette peste présente un certain retard par rapport à d'autres zones de Castille où elle apparaît en 1566. Pérez MOREDA, Vicente, La Crisis de mortalidad en la España interior. Siglos XVIe-XIXe, Madrid, Siglo XXI, 1980.

11. On dit qu'à Villadangos del Páramo quinze personnes sont décédées. À Villaviciosa de la Ribera, on déclare qu'elle sont mortes de peste, "plus de cent cinquante personnes entre grands et petits et qu'il est public et notable que plus de la moitié des habitants de tout le lieu sont morts et que presque la moitié des maisons du lieu en question sont désertées "; de leur côté, les habitants de Palacios, déclarent 20 morts victimes de la peste; par contre, dans d'autres localités, on dit après auscultation, que les malades ne présentaient aucun symptôme de ce mal. Archivo Histórico Municipal de Léon (AHML), C. $128 / 93$.

12. Voir FERNÁNDEZ VARGAS, Valentina, La población de León en el siglo XVI, Madrid, Estudios de Historia Social, Económica y Demográfica de España, Madrid, 1968, p. 161-163. En 1561 la ville de Léon comptait 997 habitants et en 15751274 . Quant à la fiabilité de ces recensements, voir RuBio PÉREZ, Laureano Manuel, León 1751 ... op. cit., p. 49 et sq.

13. Les crises agraires sont parfaitement documentées pour ces dates dans la province de Léon. RuBio Pérez, Laureano, La Bañeza y su Tierra, 1650-1850. Un modelo de sociedad rural leonesa, León, Université de Léon, 1987 et Pérez Álvarez, María José, La Montaña 
ont affecté toute la province. En 1591, les habitants de quelques localités voisines s'adressaient au conseil municipal de Léon pour qu'il prenne des mesures propres à mettre fin à la grave pénurie de pain dont ils souffraient. En $1596^{14}$, le conseil municipal a sollicité un prêt de 12000 ducas pour acheter du pain et « ravitailler le grenier et la halle de la ville, ainsi que la terre et les montagnes du Royaume de Léon ». En 1599, il s'endette de nouveau de 2000 ducas pour le même motif ${ }^{15}$. Ces crises frumentaires ont été éclipsées par les épisodes de peste ${ }^{16}$ : les habitants ont été victimes de la grande "peste atlantique " qui frappe la Péninsule ibérique à cette période ${ }^{17}$.

Malgré le creux qu'enregistre la courbe, on ne peut pas affirmer que ces incidents, pourtant d'une grande virulence, ont complètement ébranlé la base démographique de la ville. La conséquence la plus évidente a été l'érosion des gains de population accumulés progressivement jusqu'au milieu du siècle, ramenant de nouveau la population à des niveaux similaires à ceux du départ. De fait, quoiqu'en 1591, l'une des années les plus basses concernant les baptêmes, le nombre des habitants ait diminué d'un tiers par rapport à 1575 et son volume était très similaire à celui de 1555 .

Jusqu'à présent, nous avons étudié les crises à l'aide des seuls actes de baptême. À partir de 1621 nous disposons d'un enregistrement continu de décès, ce qui facilite l'identification de la chronologie et de l'intensité des épisodes dépressionnaires. En premier lieu, nous constatons que les courbes représentant les moyennes mobiles de la natalité et de la mortalité s'entrecroisent en décrivant des trajectoires inverses, ce qui confirme la maxime bien connue selon laquelle une crise de mortalité a des effets négatifs sur la natalité.

Les années vingt du XVII ${ }^{e}$ siècle débutent avec des chiffres de mortalité et de natalité situés à des niveaux relativement bas, prolongeant la situation catastrophique des années quatre-vingt-dix du XVI ${ }^{\mathrm{e}}$ siècle $^{18}$. À partir de ce moment, on peut identifier nettement les phases qui ont eu les pires conséquences démographiques sur la ville de Léon. Nous observons trois grands pics de mortalité (graphique $\mathrm{n}^{\circ} 2$ ).

noroccidental leonesa en la Edad Moderna, Léon, Université de Léon, 1996. Certains auteurs considèrent que la mortalité était plus liée à la maladie qu'exclusivement à la faim. CotTS, Susan y VAN DE VALLE, Etienne, "Nutrición, mortalidad y tamaño de la población: el tribunal de última instancia de Malthus ", dans ROTBERG, Robert I. y RABB, Theodore K., El hambre en la historia, Madrid, Siglo XXI, 1990, p. 7-30.

14. AHML, C. 43

15. AHML, Idem

16. En 1599 le Conseil municipal de Léon a convenu de concéder une aumône de cent ducas aux moines du couvent de Saint-François " vu l'extrême nécessité qu'il a eu et a à cause de la grande maladie qu'a subie cette ville et royaume de Léon, qu'est la maladie de la peste et que plusieurs des moines de son couvent l'ont attrapé au moment de confesser, administrer les derniers sacrements et aider les malades de cette ville à bien mourir ». AHML, C. 41.

17. Pérez García, José Manuel, «Demografía leonesa ... ", op. cit., p. 193. Pour l'Espagne, voir VINCENT, Bernard, « La peste Atlántica de 1596-1602 ", Asclepio, 28, 1976, p. 5-25, p. 28

18. Voir RuBio PÉREZ, Laureano M., León 1751..., op. cit., p. 58. 

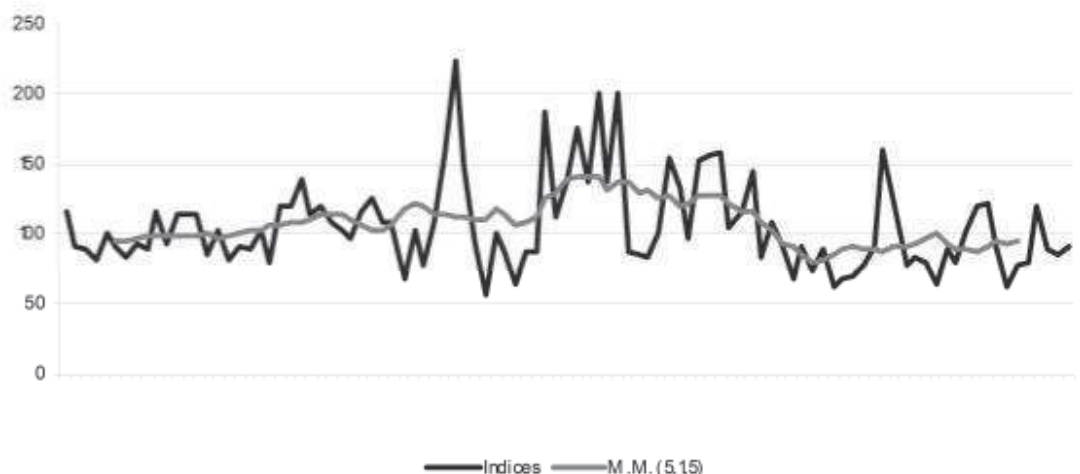

Graphique 2 - Évolution des baptêmes de la ville de León de 1620 à 1680



La première surmortalité commence au début des années quarante du $\mathrm{XVII}^{\mathrm{e}}$ siècle et culmine pendant la décennie suivante. Il ne semble pas qu'elle soit en relation avec l'épidémie apparue à Valence en 1647-1652 mais qui ne s'est pas propagée dans la Meseta grâce à l'efficacité des cordons sanitaires mis en place ${ }^{19}$. En revanche, la famine survenue pendant l'hiver de 1659-1660 20 a provoqué une crise locale qui a pesé sur les décès dans la ville de Léon.

19. NADAL, Jordi, La población española (siglos XVI al XX), Barcelone, Ariel, 1996.

20. En février 1660 , on avait déjà réalisé plusieurs prières publiques « il y a plus de 60 jours qu'il a seulement neigé, les champs sont couverts de neiges et de verglas [...] sans qu'il y ait des signes d'amélioration ». En novembre, le conseil municipal s'est vu obligé de solliciter un prêt de 8000 ducas pour acheter 1000 tonnes de pains pour la halle " dû à la stérilité de la récolte ". AHML, C. 54. 
Après une brève accalmie, apparaît la deuxième phase aiguë de mortalité. Elle a commencé à la fin des années $1670^{21}$ à la suite des difficultés agraires qui ont affecté la Couronne de Castille et, avec de brèves interruptions, a continué pendant les années 1690. Ainsi, par exemple, en 1683 les habitants de Léon ont eu recours à la médiation du Ciel avec, entre autres, la sortie en procession de la Virgen de Camino et l'ouverture du reliquaire de San Isidoro pour implorer la venue de la pluie et sauver les récoltes. Ces efforts s'avérèrent infructueux ${ }^{22}$. Le binôme classique ${ }^{23}$ famines ${ }^{24}$-maladies ${ }^{25}$ réapparaît. Dans le cas présent, il s'agit d'une poussée de " typhoïde ", liée à la pénurie d'aliments, comme celle constatée à Léon. Les années 1690 ont été particulièrement difficiles dans cette ville à cause du grand afflux de pauvres qui ont échoué là à la recherche d'aliments et à cause de l'absence d'infrastructures adéquates pour pouvoir faire front à cette " invasion ". On manquait cruellement de médecins et il fallut aménager de nouveaux lieux pour les enterrements ${ }^{26}$. La population urbaine a été alors confrontée à sutuation d'insalubrité ${ }^{27}$ dont elle a été à son tour

21. KAMEN, Henry, « The decline of Castille: the last crisis », Economic History Review, 17, 1964, p. 63-76. Cet auteur fait référence à une peste généralisée pendant les années 1680 qui a affecté aussi la ville de Léon comme le mentionnent les actes municipaux.

22. Nombreux sont les villageois qui se rendent à la halle de la ville de Léon afin de trouver un remède aux famines qui les oppressaient. Au mois de mai 1684, les soeurs du couvent de la Concepción demandent 30 tonnes de pain " pour pouvoir alimenter les religieuses dû à la nécessité dans laquelle elles se retrouvent pour ne pas avoir bien touché leurs salaires... ». Face à l'avalanche de pétitions, la ville ne pouvait pas s'occuper de toutes. AHML, C. 59.

23. Voir Martín García, Alfredo, Demografía y comportamientos demográficos en la Galicia Moderna. La villa del Ferrol y su tierra, siglos XVIXIX, Léon, Université de Léon, 2005, p. 202.

24. Ces crises sont connues dans la province de Léon, dans La Bañeza, el Bierzo et la Montaña du Nord-Ouest. RuBIo PÉREZ, Laureano, La Bañeza y su Tierra ... op. cit. BARTOLOMÉ BARTOLOMÉ, Juan Manuel, Vinos y viticultores en el Bierzo. Sociedad y estructuras económicas durante el s. XVIII, Léon, Université de Léon, 1996, y PÉREZ ÁlVAREZ, María José, La montaña noroccidental leonesa... op. cit. À Léon, on trouve des références constantes à ces famines dans les actes municipaux, où on mentionne que la ville, comme il y a cent ans, se trouve saturée de campagnards qui, provenant de la Montaña et de la Principauté des Asturies; ils cherchent à assouvir leur faim à León, " [... ] plusieurs viennent malades si bien qu'à l'hôpital il n'y a pas de lit pour les soigner ". AHML, C. 63.

25 . En 1693, le conseil municipal dit que dans la ville il y a plus de deux cents malades sans recenser " ceux qu'il y a dans les hôpitaux et d'autres maisons particulières ". AHML, C. 61.

26. La maladie a causé tant de morts qu'il n'y avait pas de lieu pour enterrer les défunts " comme le champ Malvar, où on enterre les pauvres de l'hôpital est petit et la nouvelle affluence de pauvres arrivés à la Principauté des Asturies, alors, il n'y a pas que l'Hôpital Saint-Antoine qui est plein [...] et chaque jour on enterre plusieurs cadavres et, pour le nombre mentionné, il n'y pas à présent où construire des tombes, et pour éviter les dégâts qui peuvent en surgir, ils ont décidé qu'il serait convenable de faire participer l'évêque, pour qu'il ait à bien bénir un autre pré pour pouvoir y enterrer les pauvres ", AHML, C 61.

27. "C'est la tristesse, tous les jours, de les voir mourir dans les rues et les odeurs qu'ils produisent, aussi bien dans les temples, les places et les rues que dans les maisons dans lesquelles ils entrent. Ce sont des causes à prendre en compte; il est rare de trouver une 
victime. Toutes ces difficultés se sont concentrées principalement en 1693, se répétant, avec moins d'acuité, en 1699.

Une fois terminée la crise de la fin du XVII siècle, on entre dans une période relativement tranquille qui dure jusqu'à la fin du XVIII siècle. Les registres municipaux ${ }^{28}$ montrent néanmoins que les grains vinrent parfois à manquer ${ }^{29}$, comme cela s'est produit en $1710,1749^{30}$ et 1753 . Cette relative tranquillité a permis d'atteindre de nouveau au milieu du XvIII ${ }^{\mathrm{e}}$ siècle les taux de natalité du milieu du Xvile siècle.

La troisième période de récession démographique a commencé à la fin du siècle. En 1785 déjà, on prit des mesures de prévention pour couper court à une épidémie de fièvre tierce ou paludisme ${ }^{31}$ qui s'annonçait. Entre autres avertissements, on recommande de s'approvisionner en quinquina ${ }^{32}$ et de nettoyer les lagunes et les endroits où stagnaient les eaux ${ }^{33}$. Les cinq premières années du XIX ${ }^{\mathrm{e}}$ siècle $^{34}$, furent particulièrement meurtrières, avec le plus fort taux de mortalité atteint en $1804^{35}$. Deux ans avant, la typhoïde ${ }^{36}$

maison, parmi celles qui les ont recueillis, où il n'y a pas eu de graves maladies; et si on ne les admet pas, ils s'installent dans les portails où on retrouve plusieurs d'entre eux morts [...]. Cela devient nécessaire de les obliger à sortir de la ville, cela est une opportunité pour eux et une tranquillité ". "Plusieurs d'entre eux apparaissent morts, causant de mauvaises odeurs et ils finiront par causer des maladies aux habitants durant l'été et l'automne ". AHML, C. 61.

28. AHML, C. 71

29. Durant les périodes de difficultés, le recours aux médiations religieuses pour " calmer la colère de Dieu " et obtenir la fin des châtiments était une pratique habituelle. Une des causes considérées comme ayant provoqué la calamité est "le péché des hommes ". Voir MAISo GonZÁleZ, Jesús, La Peste aragonesa de 1648-1654. Zaragosse, Université de Zaragosse, 1982 p. 34 et 58; Ballesteros Rodríguez, Juan, La peste en Córdoba, Cordoue, Diputación Provincial, 1982, p. 69.

30. Ce pic coïncide avec la crise de subsistance qui a eu lieu à Burgos. Voir SÁrnz CASADo, José Luís y SANZ DE HiguERA, Francisco Javier, "Evolución demográfica del Partido de Candemuño (1700-1850). Apuntes a la mortalidad Catastrófica ", El Pasado Histórico de Castilla y León, Vol. II, Valladolid, Junta de Castilla y León, Consejería de Educación y Cultura, 1983, p. 355-379, p. 359.

31. Cet épisode entre dans un contexte beaucoup plus ample; pendant les années quatre-vingt, le paludisme s'est étendu dans le pays, provocant une grande mortalité. Voir Alberola RomÁ, Armando y Bernabé GIL, David, " Tercianas y calenturas en tierras meridionales valenciana : una aproximación a la realidad médica y social del siglo XVIII ", Revista de Historia Moderna, 17, 1998-1999, p. 95-112, p. 102.

32. Voir Alberola Romá, Armando y Bernabé Gil, David, Ibidem, p. 110 et sq.

33. AHML, C. 77.

34. Concernant l'incidence de cette étape négative sur la principauté des Asturies voisine, voir Moro José María, Las epidemias de cólera en la Asturias del siglo XIX, Oviedo, Université d'Oviedo, 2003.

35. Au mois d'octobre 1804 , le médecin de la ville informe que "les fièvres récurentes et les tierces pernicieuses depuis juillet, ont affligé la plupart des citoyens ". Comme cause possible, il signale : " la grande quantité de vapeurs septiques et de miasmes contagieux des vêtements impurs et déguenillés des corps maladifs d'infinité de mendiants qui se préparent à entrer dans la ville ". AHML, C. 80 .

36. « [...] Les malades qui soufrent de typhoïde sortent de l'hôpital sans compléter leurs soins dans la salle de convalescence, pour cette raison ils transmettent leur maladie aux citoyens ". La Mairie a convenu, à ce moment-là, d'interdire d'accorder aux malades la 
avait frappé avec virulence la ville; cela a continué pendant les deux années suivantes. En 1805, une épidémie de variole affecta la ville de Léon et les villages voisins. On vaccina 212 personnes et un arrêté fut publié invitant tous ceux qui voulaient s'immuniser à se rendre chez le médecin titulaire de la ville. Pendant les mois de l'été et de l'automne de la même année, la teigne se propagea ${ }^{37}$. L'hôpital de Saint-Antoine et le conseil municipal se révélèrent incapables de s'occuper de tous les mendiants malades qui affluèrent vers la ville ${ }^{38}$, d'autant que l'épidémie touchait aussi la prison ${ }^{39}$. À l'occasion de ce cycle de récession, la maladie a été une fois encore précédée par le manque de grains, mais dans ce cas, les mauvaises récoltes de 1801 n'étaient pas les seules causes de la famine. La ponction de grains pour alimenter l'armée pendant la guerre au Portugal y a aussi contribué. Tout cela a fait que le prix de ce produit s'est accru dans la ville ${ }^{40}$. L'incidence de cette grande crise dans le territoire espagnol est bien connue ${ }^{41}$ et, vu son intensité, il n'est pas surprenant qu'on la compare avec celle qui eut lieu à la fin du Xvi ${ }^{\mathrm{e}}$ siècle.

Une fois surmontée l'instabilité des débuts du XIX siècle, la ville de Léon subit de nouveau, avec moins d'intensité, les effets de la guerre d'indépendance et des graves difficultés politiques et économiques surgies au début des années 1820. En 1820, il y eut un nouvel épisode de variole ${ }^{42}$, quoique sans la virulence des précédents. Au début de la décennie suivante, la ville est menacée par le choléra morbus, qui s'est déclaré dans la province pour la première fois en $1834^{43}$ dans la localité de Molinaseca. À partir de ce moment, le bilan entre la natalité et la mortalité des adultes ${ }^{44}$ enregistrées dans les paroisses de la ville de Léon est positif, ce qui indique l'absence de crise et a des répercussions positives sur la croissance de la population.

certification de guérison jusqu'à ce qu'on ait la certitude qu'ils ne sont plus contagieux. AHML, C. 81

37. On a ordonné à ce moment que " tous ceux qui souffrent de la maladie de la teigne doivent se présenter pour être soignés dans la salle installée à cet effet dans la Fábrica Vieja, où on donne aux pauvres, au moment de sortir, de nouveaux vêtements et leur enlève les vêtements qu'ils portaient à l'entrée pour éviter la contagion ". AHML, C. 81. 38. Archivo de la Catedral de León (ACL), Leg. N $^{\circ} 10052$.

39. AHML, C. 80

40. AHML, C. 79.

41. ReHER, David-Sven, «La crisis de 1804 y sus repercusiones demográficas: Cuenca (1785-1825) ", Moneda y Crédito, 154, 1982, p. 35-72.

42. "Beaucoup de malades de la variole sortent de leurs maisons sans être complètement guéris et sans que cicatrise l'épidémie, exposant ainsi le reste des citoyens à la contagion $[\ldots]$ on décide de publier un édit pour qu'aucun de ces malades ne sorte de chez lui sans être complètement guéri ". AHML, C. 84.

43. Boletín Oficial de la Provincia, 1834. Nous serions face à une deuxième vague de choléra, des sept que spécifie Bourdelais. BouRdELAIS, Patrice, La población en Francia, siglos XVII-XIX, Mexico, Instituto Mora, 1999.

44. Si nous incluions dans ce graphique la mortalité totale de la ville, hospices et hôpitaux, le bilan aurait un signe contraire. Mais n'oublions pas que dans les deux institutions on donnait un logement à beaucoup de personnes qui ne sont pas nées à León. Voir CuBILlo de la Puente, Roberto, La ciudad de León a mediados del siglo XIX. Creación de la Escuela de Veterinaria. 1852, Léon, El autor, 2002. 
Concernant la mortalité infantile (graphique 3), on peut remarquer que la courbe évolue de façon parallèle à celle des baptêmes, avec un accroissement des décès au milieu du XVIII ${ }^{\mathrm{e}}$ siècle et une baisse au début du $x^{\mathrm{e}}{ }^{\mathrm{e}}$ siècle. Ces résultats démontrent que le taux de mortalité infantile est resté très élevé à Léon pendant toute l'époque moderne. En 186027 \% des nouveau-nés ${ }^{45}$ mouraient durant leur première année de vie. En 1884, $50,3 \%$ des enfants expiraient avant d'atteindre cinq ans ${ }^{46}$. Il ne s'agit pas d'un cas isolé au niveau de la province, puisque dans la ville de Ponferrada, en 1889, 25,25\% des enfants sont morts avant l'âge de cinq ans ${ }^{47}$. Après ces dates, la mortalité dans la ville de Léon s'est maintenue à un niveau élevé avec " le triste privilège " de se situer encore en 1905 aux premiers rangs de la mortalité en Espagne.

\section{Graphique 3 - Évolution de la mortalité infantile dans la ville de León de 1750 à 1860}


La ville de Léon et le reste des noyaux urbains de la province eurent le plus grand mal à sortir du cycle de la démographie d'Ancien Régime, et ce tard encore dans le $\mathrm{xx}^{\mathrm{e}}$ siècle. La preuve est que dans la capitale, au milieu du XIXe siècle, le taux brut de mortalité était de 43,4 \% et l'espérance de vie de 26 ans ${ }^{48}$.

45. FERNÁNDEZ ARIENZA, José, Medicina y sociedad en León durante el siglo XIX, León, MIC, 1998 , p. 53 y ss.

46. Pourcentage calculé à partir des données apportées par GARCíA PONCE DE LEÓN, Ramón, Vida y muerte en la ciudad. Topografía médica de León, León, Breviarios de la Calle del Pez, ed. 1988, p. 81-84.

47. Fernández ARIENZA, José, Medicina y sociedad..., op. cit., p. 56.

48. Ibidem, p. 53. 


\section{La réponse institutionnelle face aux agents de mortalité ordinaire et extraordinaire}

Après avoir précisé la chronologie des principales phases de la mortalité extraordinaire dans la ville de Léon, il convient de faire état à présent de l'insalubrité dans laquelle se déroulait la vie des citoyens, pour mieux comprendre l'état de vulnérabilité de la population, ainsi que pour étudier l'attitude des pouvoirs publics face au phénomène.

Bien que l'excès de mortalité dans les villes ait pu être compensé par l'immigration, les effets de ces flux n'étaient pas toujours bénéfiques. Lors des crises agricoles majeures ${ }^{49}$, la ville de Léon se retrouvait débordée par l'arrivée de personnes provenant des campagnes, ce qui constituait pour la ville un motif de préoccupation supplémentaire. Ces gens mal nourris et entassés représentaient un foyer de diffusion des maladies ${ }^{50}$. À la fin du XVII ${ }^{\mathrm{e}}$ siècle, le conseil municipal se plaignait de la présence dans les rues de " quelques lépreux et galeux qui peuvent causer des infections aux autochtones ${ }^{51}$ ". Le manque d'endroits où rassembler tous ces déshérités était un motif de préoccupation pour le conseil municipal de Léon. Par ailleurs, la ville, point de référence économique pour les groupes sociaux les plus touchés qui recherchaient la charité, ne réunissait pas les conditions d'habitabilité nécessaires pour une vie salubre. Les densités élevées de population dans certains quartiers, même dans des conditions normales, facilitaient la propagation des maladies. En plus, les mauvaises conditions d'hygiène privée et publique, dues à l'accumulation des ordures ou à la consommation d'aliments de médiocre qualité, aggravaient cette situation. Les carences urbanistiques, avec des rues étroites et désorganisées ${ }^{52}$, facilitaient l'insalubrité des quartiers et des logements. S'y ajoutait l'infrastructure urbaine précaire, caractérisée par les déficiences dans le traitement de l'eau et l'absence d'égouts jusqu'au $\mathrm{XIX}^{\mathrm{e}}$ siècle.

Si chacun de ces éléments constituait en soi un facteur d'insalubrité, leur combinaison créait un environnement malsain, très propice à l'incubation et à la propagation des maladies. Pour tous ces motifs, l'un des plus grands efforts du conseil municipal de Léon, tout au long de la période étu-

49. « [... ] l'inévitable nécessité qu'il y a de guérir et d'aider les pauvres présents dans la ville, au nom de ceux qui y ont échoué venant de ces montagnes et du Royaume de León et de la Principauté des Asturies ". AHML, C. 41.

50. LIVI-BACCI, Massimo, Ensayo sobre la historia demográfica europea. Población y alimentación en Europa. Barcelone, Ariel, 1988. Cet auteur explique comment la létalité d'une poussée épidémique avait une plus grande incidence sur les populations sousalimentées.

51. AHML, C. 63.

52. "Quant aux rues, elles sont étroites; nous ordonnons, par conséquent, que personne ne mette ni table, ni banc et ne s'adonne pas à son travail au dehors de la porte ". Arrêtés municipaux (Ordenanzas Municipales), p. 148. Les arrêtés municipaux que nous avons utilisés sont ceux publiés par RuBIo PÉREz, Laureano Manuel, Ordenanzas de León, Léon, Université de Léon, 1996. 
diée, a été de garantir l'hygiène de la ville et d'améliorer les infrastructures urbaines, avec des répercussions bénéfiques sur la santé des habitants.

Le centre urbain de Léon au XVIII ${ }^{\mathrm{e}}$ siècle était pratiquement le même que celui du bas Moyen Âge. Il s'organisait, comme nous l'avons déjà mentionné, en un lacis de rues étroites et sinueuses de tracé médiéval, peu propice à la salubrité. En 1569, dans le but de remédier à cette situation, la Couronne ordonna au corregidor de Léon de rappeler aux habitants l'obligation de respecter les normes édictées au cours des règnes antérieurs ${ }^{53}$. Mais, toutes ces mesures n'ont pas réussi à remédier aux imperfections signalées. Même le plan présenté par l'architecte de la ville le 11 février $1795^{54}$, qui suivait les modèles appliqués à Madrid et dans d'autres villes en matière de construction et démolition, n'a eu aucun effet. Si bien que, peu de temps après, au début du XIX ${ }^{\mathrm{e}}$ siècle, les médecins de la ville attribuaient une partie des maladies à " la mauvaise construction de ses rues, hautes, étroites et humides, qui ne permettent pas l'entrée du soleil ni du vent pour sécher la boue et les immondices ". Cinquante ans après, les descriptions du docteur Díez Canseco ${ }^{55}$ continuaient dans la même veine. Il nous présente une ville avec des rues étroites, des conditions hygiéniques déplorables et des logements complètement insalubres qui, avec l'humidité et le manque de lumière, se convertissent en foyers infectieux. Dans les faubourgs, les conditions de salubrité étaient pires. Non seulement des immigrés galiciens et asturiens y vivaient entassés, surtout au moment des crises, mais en plus, les conditions de salubrité étaient encore plus déplorables. Il convient de rappeler que les quartiers, situés dans la partie basse de la ville, recevaient les eaux usées provenant du centre urbain, en plus de celles de l'arrosage des prés et des vergers, ce qui facilitait la propagation des maladies contagieuses.

Les difficultés résultant de l'urbanisme pouvaient se compliquer encore plus du fait que certains habitants considéraient cet espace comme le prolongement de leurs propres maisons ou locaux. Il est fréquent de trouver dans les actes municipaux des avertissements aux habitants de la ville pour qu'ils n'y laissent pas traîner des tables, bancs, cordes ou les décombres des travaux de leurs maisons.

Nous pouvons classer les réactions institutionnelles dans la ville de Léon à l'heure de prévenir et d'affronter les maladies pendant l'époque moderne, en quatre grands domaines. Le premier fait référence à la pré-

53. Reguera Rodríguez, Antonio, La Ciudad de León. Espacios y Tiempos, Léon, Université de Léon, 1996, p. 166-167.

54. AHML, C. 78.

55. Le témoignage de Juan Madrazo, en 1871 ainsi que celui de Díez Canseco, huit ans avant, vont dans le même sens : manque d'hygiène publique et domestique à cause de la mauvaise planification urbanistique et à l'ignorance des classes populaires. Le résultat de cette conjonction a été concrétisé dans les taux élevés de mortalité. Voir DiEz CANSECO, Vicente, Viruelas y vacuna. Léon, Miñón, 1863 et HERnÁndez CARRASCO, Javier y REGUERA RodRíGuEZ, Antonio, Reforma urbana liberal e infraestructura de saneamiento. El informe de Juan Madrazo sobre León, Colegio de Arquitectos, Léon, 1987. 
occupation du conseil municipal concernant l'hygiène dans le sens le plus ample du terme : nettoyage des rues et ramassage des ordures, contrôle des aliments, etc. Cette inquiétude des autorités s'observe depuis le début du $\mathrm{XVI}^{\mathrm{e}}$ siècle. Le deuxième, dont nous devons chercher les origines dans l'hygiénisme de l'époque des Lumières, nous révèle des pouvoirs locaux très impliqués et actifs en matière sanitaire, avec l'objectif principal d'approprier et d'améliorer les infrastructures urbaines. Enfin, le troisième et le quatrième vont de pair avec les cycles épidémiologiques subis par la ville, c'est-à-dire qu'ils se mettent en marche quand les taux de mortalité dépassent les limites tolérables ou sont sur le point de le faire. Ils regroupent les mesures prises face à l'avalanche de pauvres qui affluent dans la ville et toutes les mesures prophylactiques pour éviter l'entrée des maladies contagieuses.

Les arrêtés élaborés par le conseil municipal de Léon relèvent du premier domaine d'action. Les autorités étaient conscientes du lien entre la mortalité et les déficiences environnementales. Ce code pré-hygiéniste, avec des allusions explicites à la propreté et à la santé, constitue l'origine de la réglementation urbaine environnementale durant la période qui nous intéresse. Les normes ont été rédigées en 1549, publiées en 1669 et sont restées en vigueur jusqu'en 1885, année où la mairie a élaboré une nouvelle réglementation, dans laquelle elle insiste sur la nécessité de remédier aux graves problèmes dont souffre encore la ville en matière d'hygiène publique. Les arrêtés de 1549, conjointement avec le travail du conseil qui est consigné dans les actes municipaux en réponse à toutes les questions qui se posaient au quotidien, constituent en quelque sorte les principes environnementaux de base de l'époque moderne. Conjointement à ces derniers, d'autres principes extraordinaires apparaissent chaque fois qu'il y a des poussés d'épidémies, comme par exemple le contrôle des portes de la ville ou l'établissement de nouveaux lieux d'enterrement. Ils s'inscrivent dans une politique sanitaire préventive ${ }^{56}$.

L'objectif des arrêtés en matière d'environnement et de santé préventive a été de réglementer le nettoyage de la ville afin d'améliorer les conditions de salubrité : traitement des ordures et du fumier, évacuation des matières fécales, nettoyage des rues, etc. Le contrôle du respect de cet arrêté revenait à un agent municipal (alguacil) ${ }^{57}$, chargé de veiller à l'hygiène et à la qualité des aliments qui sortaient du marché.

Certains des faits que les arrêtés municipaux ont alors tenté de corriger démontrent l'importance acquise par quelques principes pré-hygiéniques

\footnotetext{
56. Les actions municipales concernant la santé publique peuvent être considérées comme essentielles pour le progrès de la médecine préventive. SáEz GóMEZ, José Miguel y Marset CAmpos, Pedro, "Teoría académica y práctica ciudadana en el paludismo. Las causas de las enfermedades endémicas en Murcia durante el siglo XVIII desde la perspectiva de la administración local ", Aslcepio, 52, 2000, p. 167-184, p. 170.

57. Tout ce qui se réfère aux arrêtés de 1549 peut être consulté dans RuBIo PÉREz, Laureano Manuel, Ordenanzas..., op. cit.
} 
qui recherchaient l'amélioration des conditions de vie des habitants de Léon. Le concept de propreté urbaine était parfaitement enraciné dans les arrêtés élaborés dans la deuxième moitié du $\mathrm{XVI}^{\mathrm{e}}$ siècle. Les rues étaient en effet le champ d'action de plusieurs activités professionnelles des classes populaires et, par conséquent, elles se transformaient en authentiques dépôts d'ordures. Elles étaient en même temps le cadre de la vie sociale et le lieu de circulation des animaux. Dans le concept de nettoyage des rues est, en outre, inclus celui d'éviter de les salir. À partir de là, on a prêté plus d'attention à toutes les questions dérivées de la présence d'animaux dans l'espace urbain. Les fumiers, les restes d'aliments ou de littière étaient des foyers d'insalubrité. La municipalité a constamment insisté sur le nettoyage des rues tout au long de l'époque moderne ${ }^{58}$, mais les mesures législatives édictées n'étaient pas assez strictes ou bien elles n'avaient pas l'effet adéquat pour mettre fin à tous les foyers d'insalubrité.

La grande poussée de l'hygiène publique n'a eu lieu qu'avec l'avènement du mouvement des Lumières au XVIII ${ }^{\mathrm{e}}$ siècle $^{59}$. Témoignant de cette préoccupation, un accord est signé en 1756 entre le Marquis de Inicio, représentant de la ville, et quelques habitants de la banlieue pour le nettoyage des rues. Mais au vu des sources, celui-ci ne s'effectuait pas avec la périodicité et la scrupulosité nécessaires. Une solution, qui n'était pas définitive mais du moins constituait une avancée, apparaît dans le code élaboré le 5 janvier 1801, sur le modèle de celui de Madrid de 1797. Celui qui a été rédigé pour la ville de Léon ${ }^{60}$ règlemente avec beaucoup de minutie tout ce qui se réfère au nettoyage des annexes des maisons particulières ${ }^{61}$ et des édifices ecclésiastiques. Dans les deux cas, la responsabilité de maintenir propre cet espace public revient aux habitants qui, chaque jeudi après-midi, doivent se consacrer à cette besogne. Les ordures devaient être ramassées et accumulées dans un lieu ne gênant

58. Dans le but de maintenir des conditions minimales de salubrité et d'habitabilité, les Conseils municipaux de toutes les villes ont insisté sur le thème de la propreté des rues, y compris dans les époques postérieures à celle qui nous intéresse. Voir ANAUT BraVo, Sagrario, "Higiene urbana y mortalidad en Pamplona (1880-1935) ", Revista de Demografía Histórica, xx, II, 2002, p. 113-145, p. 123. Voir aussi, Cipolla, Carlo M., Contra un enemigo mortal e invisible, Barcelona, Crítica, 1993, p. 24-42.

59. Pour connaître plus à fond les préoccupations des penseurs espagnols des Lumières au sujet de la croissance démographique, la politique sanitaire et les méthodes curatives, voir BETRÁN MoyA, José Luís, Historia de las epidemias en España y sus colonias (13481919), Madrid, La Esfera de los Libros, 2006. En France on pourrait considérer l'an 1776, durant lequel est réalisée la première topographie médicale, comme le point de départ pour évaluer et corriger la santé urbaine. Voir Vigarello, Georges, Histoire des pratiques de santé, Paris, Seuil, 1990.

60. AHML, C. 79

61. «[...] pour éviter la poussière des balais, par les temps secs de l'été ou de l'hiver, il y a un risque dans ce qu'il faudrait balayer "; " [...] que personne ne secoue au balcon, de la fenêtre, des parapets ou portes de sa maison à la rue, vêtements, tapis de nattes, paillassons, ni meubles, qu'ils s'abstiennent aussi de verser ou jeter des immondices, de l'eau ou quelque autre déchet [...]"; " [...] qu'ils vident les déversoirs d'eaux sortants des maisons à travers les murs aux rues ou places ». AHML, C. 78. 
pas et le vendredi matin elles devaient être chargées dans des chariots de nettoyage.

Pour le nettoyage des lieux publics, on employait des balayeurs dont le salaire était payé, en partie, par les amendes imposées aux contrevenants à l'édit. On interdisait aussi de secouer les vêtements, les tapis et autres objets aux balcons ou de jeter des immondices par les fenêtres hors des horaires permis et sans donner de préavis. Le soin de faire respecter cette réglementation était confié à un regidor qui faisait office de " commissaire de police $^{62}$ ", assisté par un alguacil. Le fait que le conseil municipal revienne constamment sur ce thème et augmente les amendes infligées aux contrevenants démontre le peu d'effet que ces mesures hygiéniques et sanitaires avaient sur le peuple ${ }^{63}$. Ce manque d'obéissance fut sans cesse dénoncé, comme le démontre le rapport des médecins élaboré pendant l'été 1801, quand apparurent les fièvres putrides ou tierces. Ces derniers proposaient comme première mesure préventive le nettoyage des rues, l'arrosage des trottoirs, le changement du comportement des marchandes de légumes et des poissonniers ou le déplacement des dépotoirs loin de la zone habitée. Un nouvel édit fut bientôt publié, dans lequel les autorités locales tentaient d'apporter des solutions à toutes ces questions en menaçant les habitants de punitions pécuniaires. Malgré toutes les mesures coercitives, l'incorporation de l'hygiénisme dans la vie quotidienne a été très lente.

En 1790, apparaît le ramassage organisé des ordures, bien que la municipalité ait antérieurement créé un réseau de dépôts de déchets. En 1761, on demande à l'agent chargé de la police de chercher des endroits appropriés dans la ville pour déposer les déchets; il signale quatorze points, presque tous situés dans l'enceinte fortifiée. Nous devons supposer qu'après leur stockage on procédait à leur évacuation ${ }^{64}$. En 1801, le service de ramassage des ordures fut mis aux enchères et l'adjudicataire obligé de les ramasser une fois par semaine. La veille, les voisins devaient sortir leurs déchets dans la rue. Il semble que cette fréquence n'était pas appropriée puisqu'une année plus tard ${ }^{65}$, face à l'inquiétude que les ordures continuaient à provoquer chez les regidores, on en confiait le ramassage aux habitants de la banlieue les mardis et les vendredis.

62. "à sa charge seraient le soin des pavés et le nettoyage des rues ". AHML, C. 79.

63. En 1801, on se plaignait que les marchandes de légumes "ne ramassent pas les déchets qui se corrompent dans les places; on jette aussi des excréments, du fumier "; " qu'on respecte l'édit de janvier concernant la propreté et qu'on le publie de nouveau avec amendes aux contrevenants, que pour le prochain Corpus, on balaie les rues les lundis et qu'on oblige les charretiers à se déplacer avec leurs chariots sans oublier de sortir les ordures ". AHML, C. 79.

64. La façon dont cela était organisé à ce moment nous l'ignorons, comme le mentionne certain chapitre des Arrêtés, mais nous supposons qu'il devait être similaire à celui accordé en 1790. Dans la ville d'Astorga en 1519, le ramassage des ordures était déjà plus ou moins organisé. MARTín Fuertes, José Antonio, El Concejo de Astorga (siglos XIII-XVI), Astorga, Institución " Fray Bernardino de Sahagún», 2004, p. 63.

65. AHML, C. 79 
Les arrêtés municipaux ont insisté sur la nécessité d'interdire la présence d'animaux dans les rues, tout particulièrement les cochons, afin d'éviter leur divagation dans la ville. Malgré cela, en 1785, la ville était obligée de publier un nouvel édit ordonnant aux propriétaires de troupeaux de cochons de respecter les tours de rôle (veceras) ${ }^{66}$. En 1801, les médecins proposaient au conseil municipal de déplacer l'élevage des porcins dans les faubourgs pour éliminer les saletés qu'ils produisaient et, par extension, l'insalubrité. Les magistrats municipaux répondirent qu'ils ne pouvaient pas interdire aux habitants de loger ces animaux dans des espaces privés ni légiférer sur la propreté de ces endroits. Ils pouvaient seulement continuer à insister et à prendre des mesures encore plus draconiennes pour que soit respecté ce qui était déjà interdit.

En référence à la salubrité de l'air, on se borne à répéter, depuis le $\mathrm{XVI}^{\mathrm{e}}$ siècle, et sans qu'il y ait aucun changement substantiel dans la réglementation durant l'époque moderne, des mesures tendant à éviter les mauvaises odeurs. Celles-ci devaient être assez fréquentes en raison du manque d'hygiène dans les rues, des déchets organiques, de l'eau sale qu'on y jetait, en particulier celle provenant du nettoyage du poisson, des émanations des fosses d'aisance, des vapeurs de la fermentation du pain, etc. Dans ce but, les médecins ont proposé au conseil municipal, au début du XIXe siècle, de faire sortir les fours du centre urbain, mais le conseil a considéré que ces installations ne contribuaient pas à "l'infection de l'air ".

La question de santé prioritaire était l'alimentation. La politique d'intervention municipale jusqu'au milieu du $\mathrm{xx}^{\mathrm{e}}$ siècle, où le conseil a perdu ce type de compétences, s'est centrée sur le contrôle de la qualité des produits. Depuis l'époque moderne, la ville de Léon comptait un abattoir, mais celui-ci, tout comme les boucheries, ne réunissait pas les conditions sanitaires nécessaires pour garantir au consommateur la qualité des produits ${ }^{67}$. Une autre grande préoccupation des autorités, comme le prouvent les délibérations municipales, était l'approvisionnement des habitants de Léon en aliments frais. Par exemple, en 1801, on interdit la vente de vin jeune de Toro " jusqu'à ce qu'il soit épuré et avec moins de particules sulfureuses ", vu qu'il était en train de provoquer des maladies chez les habitants ${ }^{68}$. On interdit également de vendre du lard frais durant les mois d'été ou de mettre sur le poisson des produits pouvant masquer sa qualité. En plus, les abattoirs sont contrôlés pour éviter le sacrifice d'animaux malades, etc. Vu qu'il n'y avait pas de personnel qualifié jusqu'à 1799, toute l'inspection des aliments se faisait de manière empirique. À partir de cette date, l'intervention sur les différents marchés revint aux médecins et aux chirurgiens, qui assumèrent cette responsabilité jusqu'au premier tiers du $\mathrm{XIX}^{\mathrm{e}}$ siècle, avant leur remplacement par les vétérinaires ${ }^{69}$.

66. AHML, C. 77

67. Cubillo de la Puente, Roberto, La ciudad de León... op. cit., p. 47 et sq.

68. AHML, C. 79.

69. CUBiLlo de LA PuENTE, Roberto, Comer en León : un siglo de historia, 1700-1800, Léon, Université de Léon, 2000, p. 82. 
Le deuxième champ d'action était celui de l'amélioration, ou de la création dans le cas présent, d'une infrastructure urbaine nécessaire pour la salubrité publique. Une grande partie des plans d'action qui ont été réalisés dans ce domaine sont le fruit du concept hygiéniste du XvIII ${ }^{\mathrm{e}}$ siècle. Garantir un approvisionnement correct de la ville en eau était prioritaire. Les premières informations sur cette question, à l'époque moderne, datent de 1530. Cette année-là, le conseil de la cathédrale et le conseil municipal parviennent à un accord pour la conduite des eaux de la place de la Regla, ou de la Cathédrale, à la place de San Martín. Ces travaux ne sont connus que cinquante ans après, lorsque, en 1581, pour terminer de les payer la municipalité doit vendre un pré ${ }^{70}$. Jusqu'au XvIII ${ }^{\mathrm{e}}$ siècle, malgré certains projets comme celui de 1638, l'approvisionnement en eau de la ville de Léon a laissé beaucoup à désirer. Pour ce motif, pendant les dernières décennies du XVIII ${ }^{\mathrm{e}}$ siècle, le conseil municipal, conscient des dommages qui résultent pour la santé des citoyens du manque d'une canalisation correcte ${ }^{71}$, entreprend un important projet d'approvisionnement d'eau pour toute la ville. Les travaux ont été financés avec l'argent qui restait des abonnements des rentes royales qu'il gérait depuis quelques années ${ }^{72}$. En 1773 a été créé l'Assemblée des sources et canalisations (Junta de Fuentes y cañerías) ${ }^{73}$, formée par des représentants de la municipalité et du chapitre de la cathédrale. L'une des mesures prises au sein de ce projet d'assainissement de l'eau a été celle du contrôle quotidien des sources et des canalisations par un plombier, qui devait réparer n'importe quelle avarie. On désigna aussi un surveillant dont la tâche était de veiller à la propreté des sources. De plus, il était primordial de réaliser un nouveau tracé pour la conduite des eaux. La proposition en fut faite par le procureur (personero) qui, assisté par les médecins, attribuait quelques-uns des dommages subis par la santé des habitants de Léon au mauvais état dans lequel arrivait l'eau pour la consommation, portant " très salubre dans son origine".

Le conseil municipal parvint à un accord avec le chapitre de la cathédrale pour augmenter le débit d'eau potable distribuée dans la ville et pour décider à qui incombait le financement de tous les travaux de maintenance, estimés à 6000 réaux par an. Le chapitre accepta de contribuer pour 1500 réaux, le reste devant être fourni par la ville et le peuple. Par ailleurs, tous les habitants en état de payer l'eau ainsi que les couvents de réguliers pouvaient se connecter à cette canalisation générale, sachant qu'ils seraient les premiers affectés par les restrictions si le débit venait à baisser. Bien que l'approvisionnement direct en eau ne soit garanti que pour une minorité de

70. AHML, C. 714.

71. «[...] elles se détériorent de façon qu'elles portent de véritables préjudices à la santé publique comme le confirment les médecins et les chirurgiens de la ville, pour ne pas être salubres dès leurs origines ". AHML C. 707.

72. AHML, C. 714 y 715.

73. AHML, C. 707. «Pour continuer à avoir des informations au plus tôt de ce qui se passe et continuer dans une bonne ligne ce projet ". 
privilégiés, le projet permettait aussi un approvisionnement des quartiers les plus peuplés au moyen de sources publiques.

Malgré tous ces efforts, la politique hydraulique n'était pas suffisante. En 1788, le conseil municipal se plaignait du manque d'eau potable et, en 1792, les déficiences du réseau étaient soulignées dans la Gaceta Pública ${ }^{74}$. Les habitants dénonçaient le fait d'être, à plusieurs reprises, obligés de boire " de l'eau trouble et nuisible à cause de la détérioration des canalisations ». La situation ne paraît pas avoir changé en 1801, vu que dans le rapport élaboré par les médecins de la toute nouvelle Assemblée de la santé $^{75}$, on proposait de recruter des porteurs d'eau publics, en raison des mauvaises conditions dans lesquelles se trouvait l'eau des sources "pour sa dureté naturelle ". Les médecins attribuaient la mauvaise qualité de l'eau à « la malignité attrapée pendant qu'elle court à travers des armatures de plomb ".

Pendant les premiers siècles de l'époque moderne, le système de canalisation était inexistant et son développement très lent dans la ville. Jusqu'au $\mathrm{XX}^{\mathrm{e}}$ siècle, Léon n'a pas disposé d'un réseau de distribution complet ${ }^{76}$, ce qui en faisait un lieu menacé par la pestilence, surtout dans les endroits où, en l'absence de pente, les eaux résiduelles s'accumulaient. Les premiers projets pour remédier ce problème débutèrent pendant le dernier quart du siècle, mais les résultats furent très limités. Disposèrent de ce réseau d'évacuation les rues les plus propices, c'est-à-dire celles qui présentaient une pente suffisante pour l'évacuation des résidus. Le reste du maillage urbain dut attendre les deux dernières décennies du XIX ${ }^{\mathrm{e}}$ siècle; le centre de la ville ${ }^{77}$ fut alors doté d'un système d'évacuation adéquat.

Quant au pavage des rues ${ }^{78}$ et à la structure viaire ${ }^{79}$, il n'y a pas de doute sur la nécessité qu'ils devaient être en parfait état pour faciliter le déplacement des habitants, mais aussi contribuer à l'hygiène des rues et à la santé des citoyens. Les zones marécageuses étaient les responsables

74. Risco, Manuel, Historia de la ciudad y corte de Leon, y de sus reyes, Valladolid, Pentalfa, 1989, p. 129.

75. Sa création obéit à un ordre royal du 30 septembre 1800. RoDRíGuEZ OcaÑa, Esteban, " El resguardo de la salud. Organización Sanitaria en el siglo xviII ", Dynamis, 7-8, 1987-1988, p. 145-170, p. 150. À Léon, elle a été créée le 26 septembre 1800, presque dix ans après celle de Valladolid. MERCHÁn Fernandez, A. Carlos, Gobierno Municipal y Administración Local en la España del Antiguo Régimen, Madrid, Tecnos, 1988, p. 286.

76. Les premiers progrès ont eu déjà lieu au début du siècle précédent; au mois de mai 1801, ont été terminés les travaux de la canalisation de la rue de San Pedro de los Huertos. AHML, C. 79.

77. Dans la rue Serrano, la canalisation a été réalisée en 1889, dans la rue del Cid en 1894 et, dans la rue Varillas en 1897. La canalisation des rues La Rua, Conde Rebolledo y San Macelo a été mise aux enchères en 1886. AHML, C. 724.

78. Au sujet de la place du Conde de Luna, on la décrit comme: « dépavée, pleines de trous, de flaques d'eaux de pluies accumulées et contaminées ". AHML, C. 79.

79. « Les routes qui se dirigent vers cette ville, à Astorga et à des endroits de San Andrés et Villabalter sont inondées d'eau [...] à cause de l'abondance et du dérèglement de l'arrosage des prés ». AHPL, C. 79. 
des fièvres « tierces " et " quartes " (paludisme), particulièrement pendant les mois d'été ${ }^{80}$. Les travaux de maintenance devaient avoir lieu avec une certaine fréquence. Par exemple, en 1749, la municipalité dépensa 239 réaux pour le pavage et le nettoyage des rues et 500 en 1750 . Il semble qu'à la fin du siècle on ait réalisé une réforme plus profonde, à en juger par les commentaires de Manuel Risco qui parle du "nouveau et curieux pavage " des rues, financé sur fonds municipaux. À partir de 1771, et après la publication d'un ordre royal qui permettait à la ville d'investir 8000 réaux par an dans la réparation et l'assainissement des rues " pour qu'elles soient le plus propre possible ", ce labeur a été intensifié. En 1801, le conseil municipal se vantait du bon état des voies et des petites places de la ville, ainsi que du pavage de " presque tous les trottoirs". Cependant, ce panorama positif était assombri par le manque d'hygiène et de respect de l'espace public de la part des habitants, converti qu'il était par ces derniers en " centres d'immondices qui, non seulement affectent la santé des gens, mais les dérangent et les offusquent ". D'autre part, le parfait état des voies et passages qu'on mentionne à certains moments ne durait pas longtemps, du fait du passage incessant des chariots et des charrettes ${ }^{81}$, des travaux des particuliers et de l'absence de personnel qualifié pour veiller à leur conservation. C'est ce que dénonçait en 1802 le député du peuple. Pour cette raison, cette année-là, on recruta un cantonnier chargé de ces besognes. Malgré tous ces progrès, vers la fin de l'année 1850, le voyageur allemand A. Ziegler décrit un pavage qui "laisse beaucoup à désirer ", sauf dans deux rues, à cause d'un revêtement fragile du fait de l'emploi de cailloux et de gravillons. Les témoignages de F. Gadow, F. Elliot et C. Daviller vont dans le même sens. Ils décrivent Léon comme une ville lugubre, avec des rues semées de nids-depoule et d'ornières terribles ${ }^{82}$.

Une autre question qui devaient être traitées au moment de veiller à la santé et à l'hygiène publiques était celle des cimetières, en raison des effets négatifs que cela pouvait avoir sur la ville, mais on ne parvint pas à des résultats positifs pendant le xvIII siècle. En plus des inhumations dans les églises ${ }^{83}$, il y avait des cimetières où l'on enterrait les indigents (malvares); ils se trouvaient saturés au moment de grandes crises de subsistance,

80. Voir BERnABÉ GIL, David, « Insalubridad y bonificaciones de almarjales en el Bajo Segura antes de las Pías fundaciones de Belluga ", Revista de Historia Moderna, 17, 19981999, p. 45-72.

81. Dans ce sens, en 1771, on avait publié un édit par lequel on interdisait aux meuniers de transporter plus de 4 tonnes de blé dans leurs chariots, vu que s'ils transportaient plus de charge, ils détruiraient le pavage. Les amendes pour contravention augmentaient en fonction du niveau de désobéissance. AHML, C. 75.

82. CASAdo Lobato, Concha y Carreira Vérez, Antonio, Viajeros por León. Siglos XII-XIX, Léon, Santiago García, 1992, p. 79 et sq.

83. Tout individu pouvait choisir comme lieu d'enterrement le lieu sacré, à cause de la conviction que ce choix lui apporterait plus de bénéfices spirituels et renforcerait l'efficacité des suffrages. GRANJEL, Mercedes y CARRERAS PANCHON, Antonio, "Extremadura y el debate sobre la creación de cementerios: un problema de salud pública en la ilustración ", Norba. Revista de Historia, 17, 2004, p. 69-91, p. 76. 
quand la ville, non seulement devait affronter les problèmes causés par ses propres pauvres et malades, mais aussi ceux provoqués par les migrants venus des campagnes et de l'extérieur de la province pour fuir la famine et qui tombaient dans un piège encore plus mortel, celui de la ville.

L'ordre de déplacer les cimetières hors des agglomérations a été approuvé par Charles III en $1786^{84}$, dans le contexte hygiéniste des Lumières qui s'étendait en Europe, et ratifié par les Cortes de $1813^{85}$. Il y eut à ce moment un problème difficile à résoudre : l'affrontement entre le pouvoir civil et le pouvoir ecclésiastique au moment de décider de la construction du cimetière et d'attribuer la juridiction sur celui-ci ${ }^{86}$. À Léon, l'une des premières références relatives à ce sujet est antérieure à 1786. En 1783, quand l'hôpital de Saint-Antoine entreprit la construction d'un cimetière des pauvres (malvar) à l'arrière du bâtiment, le conseil tenta d'arrêter les travaux. Treize ans plus tard, le corregidor demanda à l'évêque et au conseil municipal que soit supprimé le cimetière en question, où étaient enterrés de nombreux pauvres et des soldats du régiment de Léon "par précaution contre les préjudices qui peuvent répercuter sur la santé publique " et qu'on le déplace à l'endroit que les médecins avaient signalé ${ }^{87}$. D'autre part, en 1804, quand la ville fut touchée par une grande poussée de typhoïde ${ }^{88}$, l'évêque trouva une formule intermédiaire : obéir à l'ordre de Charles III mais laisser de côté le pouvoir civil. Le prélat ordonna que les cadavres soient déplacés dans les ermitages qui se trouvaient hors des zones habitées et qu'on aménage un espace à cette fin dans les alentours de ceuxci ${ }^{89}$. C'était, par conséquent, une solution unilatérale, mais nous ne devons pas pour autant sous-estimer sa bonne volonté. La réponse définitive au Brevet du Roi de 1787 eut lieu en $1809^{90}$. Un édit municipal interdisait catégoriquement les enterrements dans les églises et ordonnait de construire

84. Cette date coïncide avec l'exhumation des cadavres du cimetière parisien des Saints Innocents, qui a été déplacé. Voir Vigarello, Georges, Histoire des pratiques... op. cit., p. 185.

85. Entre les deux il y eut toute une série d'ordres qui rappelaient aux institutions la nécessité de construire des cimetières hors de la ville. Voir SANTOJA, José Luís, " La construcción de cementerios extramuros : Un aspecto de la lucha contra la mortalidad en el Antiguo Régimen ", Revista de Historia Moderna, 17, 1998-1999, pp. 33-44, p. 34 et sq.

86. GonZÁlEz DíEZ, Alicia, "El cementerio español de los siglos XVIII y XIX", Archivo Español de Arte, 171, 1970, 289-320, p. 290 sq.

87. AHML, C. 78.

88. Après plusieurs tentatives antérieures également à Cordoue, le point d'inflexion est marqué par les épidémies des cinq premières années du XIX ${ }^{\mathrm{e}}$ siècle. À ce moment-là, la ville a décidé sérieusement de créer un cimetière hors de l'enceinte urbaine. GómEZ NAVARRo, María Soledad, "La construcción de cementerios en la provincia de Córdoba, 1787-1833 ", Una arquitectura para la muerte. Actas del I Encuentro internacional sobre cementerios contemporáneos, Sevilla, Consejería de Obras Públicas y Transportes, 1993, p. $399-405$, p. 400 .

89. HERnÁNDEZ CARRASCO, Javier y REgUERA RodRÍGUEZ, Antonio, Reforma urbana liberal... op. cit., p. 30.

90. Ce retard par rapport à l'ordre de Charles III était très habituel en Espagne. Voir SANTOJA, José Luís, "La construcción de cementerios extramuros... ", loc. cit., p. 34-44. 
un cimetière public à un kilomètre de distance du noyau urbain. En raison du mauvais choix de l'endroit pour implanter le cimetière, la ville continua à supporter les effluves qui émanaient de la putréfaction des cadavres. Comble de malheur, le nouveau cimetière était très proche de l'une des sources qui approvisionnaient la ville en eau potable ${ }^{91}$. Bien que tout parût avoir été résolu dans la première décennie du XIX siècle, en 1884, il y avait encore quatre cimetières conventuels dans le périmètre urbain ${ }^{92}$.

Parmi les déficiences urbanistiques, il faut inclure l'absence d'éclairage public des rues, l'obscurité de la nuit étant très propice pour commettre des délits. L'une des mesures préventives dictées par le pouvoir local a été celle d'ordonner que tous les habitants qui se déplaçaient la nuit disposeraient d'un éclairage et que ne se formaient pas des groupes de personnes à des heures intempestives. Un arrêté municipal du 13 novembre 1801 insiste sur cette question après que l'on ait constaté " le manque de respect depuis quelque temps ${ }^{93}$ ». Cette réglementation prend place dans un contexte problématique pour la ville, qui se trouvait submergée par l'arrivée d'un nombre élevé de pauvres et d'étrangers qui, dans quelques cas, pouvaient représenter un danger. Les peines sévères ${ }^{94}$ avec lesquelles on punissait ceux qui violaient l'arrêté témoignent du danger que pouvait représenter le fait de circuler dans les rues de Léon pendant la nuit.

Dans le but de favoriser la cohabitation pacifique, un édit a été publié par ordre du corregidor, le 23 juillet 1801, interdisant aux habitants de la ville et aux gens de passage d'entrer boire dans les tavernes ${ }^{95}$. Pour l'éviter, les tables seraient placées de façon à occuper toute l'entrée, en plus, les tavernes devraient être fermées quand sonnerait la cloche. À partir de cet instant, on ne permettait plus de servir de l'eau-de-vie et des liqueurs qu'aux personnes qui justifiaient en avoir besoin au moyen une ordonnance signée par le médecin ou le chirurgien. Cette mesure a été rapidement critiquée par D. Vicente Alonso Moreno et D. José Herrera, qui avaient l'adjudication de la vente de l'eau-de-vie. Sachant que tous les deux étaient regidores de la ville et tenant compte des pressions qu'ils ont exercées, on n'a pas tardé à révoquer l'édit.

En 1802, une nouvelle proposition a été présentée afin de doter la ville d'une infrastructure urbaine complète, mais il semble qu'elle n'ait trouvé aucun écho. Il s'agissait d'installer des lavoirs et des séchoirs publics pour que les femmes " puissent laver le linge [...] sans qu'elle risquent d'incen-

91. García Y Ponce De León, Ramón, Vida y muerte en la ciudad (Topografía Médica de León), Léon, Breviarios de la Calle el Pez, 1988, p. 46.

92. AHML, C. 750.

93. AHML, C. 79.

94. Les amendes pour les contrevenants sont de deux ducas la première fois, quatre la deuxième et huit les suivantes, plus quinze jours de prison.

95. Vu que " elles sont fréquentées par des artisans et des gens misérables qui ont l'habitude de rester là pendant beaucoup de temps, même les jours de travail, jusqu'à se saoûler, ce qui provoque, des fois, des disputes, du vacarme et des dégâts, et plus grands dans leurs maisons et à leurs familles ". AHML, C. 71. 
dier leurs maisons... comme c'est le cas plusieurs fois au moment de chauffer l'eau pour la lessive $[\ldots]^{96}$ ".

Concernant le troisième bloc de mesures, les actes municipaux sont émaillés de témoignages qui font référence à des étrangers pauvres ${ }^{97}$ qui arrivent en ville pendant les époques d'insécurité et les fréquentes périodes critiques $^{98}$. Ce qui préoccupait le plus les représentants de la ville n'était pas seulement qu'on était dans l'impossibilité de les nourrir et de s'occuper d'eux, mais aussi qu'ils se transformaient en agents de propagation de maladies infectieuses du fait du manque d'hygiène, de leur dénutrition et de leur entassement. Afin de résoudre tous ces problèmes, l'État a élaboré les lois des pauvres. Les pouvoirs publics et religieux, en étroite collaboration, ont fondé à la fin du XVII ${ }^{\mathrm{e}}$ siècle, un hospice pour nécessiteux et un refuge pour ceux qui ne pouvaient pas continuer leur voyage par manque d'aliments. Cette entente entre les deux institutions découlait de l'impossibilité pour chacune d'affronter seule le problème par manque de moyens financiers. De plus, le chapitre cathédral s'était offert à aménager deux salles dans l'hôpital Saint-Antoine, l'une pour soigner les pauvres malades et l'autre, au quatrième étage, au préalable occupée par les administratifs, pour héberger les convalescents ${ }^{99}$. L' "Hospice pour Pauvres ${ }^{100}$ ", qui avait une capacité d'accueil de 10 à 12 personnes, annonce la Maison de la charité (Casa de Mendicidad) fondée à Léon en 1855.

Concernant les mesures prophylactiques à caractère extraordinaire, on constate que la réglementation qui émanait du conseil municipal de Léon est pratiquement la même à trois moments de danger potentiel d'épidémie (1587, 1599 et les dernières années du XVII ${ }^{\mathrm{e}}$ siècle). Cela nous amène à affirmer que, face à ces épisodes, le dispositif institutionnel est en place plus ou moins depuis 1587. À partir de ce moment-là, on s'est seulement borné à nuancer ou à corriger ces mesures afin de les adapter aux besoins. Une fois connue la présence de la maladie dans des zones proches, tout un mécanisme de prophylaxie se mettait en marche. Le point central de cette législation fut la création de cordons sanitaires ${ }^{101}$ qui isolaient la ville. Leur

96. AHML, C. 79. Ce sont des raisons très distinctes de celles qui incitent les Majorquins et les Catalans à la création de ces lavoirs. Voir SALAS VIVES, Pere, " La política sanitaria en la primera mitad del siglo XIX ", Revista de Demografía Histórica, xx-2, 2002, p. 53-97, p. 76 et $s q$.; IRLES VICENTE, María del Carmen, " Proyectos y realidades: higiene y salud pública en la Cataluña de finales del setecientos ", Revista de Historia Moderna, 17, 1998-1999, p. 147-166, p. 155 .

97. " [...] à cause de la grande quantité de pauvres qu'il y a dans la ville augmentant chaque jour, de la stérilité qu'il y a et qu'il y a eu dans la Principauté des Asturies [...]. Beaucoup des pauvres sont morts et pour ceux qui vont à l'hôpital, la plupart meurent là-bas et quelques-uns viennent avec la lèpre et marchant dans les rues ". AHML, C. 63. 98. LÁzaro Ruíz, Mercedes y GuRria García, Pedro A., " Las crisis de mortalidad en La Rioja ", Revista de Demografía Histórica, Vol. 7- n 1, 1989, p. 31-46, p. 44.

99. AHML, C 61.

100. AHML, C. 43

101. Il s'agit d'un comportement type appliqué dans tout le territoire européen et national. À titre d'exemple, voir les travaux suivants : BETRÁn MoYA, José Luís, La peste en el 
efficacité reposait sur le contrôle des entrées que les gardiens imposaient aux étrangers. En $1804^{102}$, face à la tournure que prenaient les évènements, le médecin de Léon, M. Manuel Risco Merino proposa de prendre des mesures énergiques pour interdire l'entrée dans la ville aux mendiants coupables, d'après lui, de l'état de maladie dont souffrait celle-ci. Les moyens d'action étaient au nombre de deux, d'une part, installer deux gardiens à chacune des portes d'accès à la ville, pendant que trois autres la parcouraient pour expulser les infiltrés et, d'autre part, face à la proximité de la foire de Toussaint, demander à ceux qui y assisteraient un passeport et certificat de santé en "interdisant l'entrée à celui qui ne l'apporte pas ". Cependant, il est évident que, malgré tous ces cordons et la vigilance, les contagions ont réussi à entrer dans la ville et à causer des ravages ${ }^{103}$.

Au début du XVII ${ }^{\mathrm{e}}$ siècle, l'État a institutionnalisé l'Assemblée de santé (Junta de Salud), qui avait des représentants dans les différentes villes du royaume, à travers lesquels il centralisait l'information nécessaire pour prévoir les épisodes de peste. En 1720, cette assemblée s'est convertie en l'Assemblée suprême de santé " La Junta Suprema de Sanidad ${ }^{104}$ ", avec des délégations dans toutes les provinces, qui se sont constituées à des dates différentes. Celle de la ville de Léon fut créée le 26 septembre $1800^{105}$ et cette assemblée s'est convertie au Xxx ${ }^{\mathrm{e}}$ siècle, avec l'apparition postérieure du Journal officiel de la province (Boletín Oficial de la Provincia), en l'instrument de diffusion de la politique sanitaire officielle.

L'assemblée suprême de la santé a fonctionné jusqu'à 1847, année où elle fut remplacée par la Direction générale de la santé (Dirección General de Sanidad). L'une de ses actions en 1800 a consisté à avertir les institutions de justice dans toute la province de la présence d'une poussé épidémique dans le sud de la Péninsule, afin que soient prises les mesures opportunes pour éviter sa propagation. L'information arrivait aux chefs-lieux d'arrondissement. De là, elle était répercutée à chacune des entités qui en faisaient partie, les représentants devaient signer un document dans lequel ils affir-

Barcelona de los Austrias, Lleida, Milenio, 1996; BAlLeSTERos RodríGUEZ, Juan, 1982; CiPOLLA, Carlo Maria, Contra un enemigo mortal e invisible, Crítica, Barcelone, 1993; LÁzARo Ruíz, Mercedes y GuRRIA GARCía, Pedro A., "Las crisis de mortalidad... ", loc. cit.

102. AHML, C. 82.

103. LÁZARo Rúz, Mercedes y Gurria García, Pedro A., " Las crisis de mortalidad... ", loc. cit., p. 41-66. Ces auteurs ont trouvé des références à la violation de l'Arrêté et ils considèrent qu'une fois passé le premier impact les contrôles se relâchent petit à petit.

104. Concernant les avatars de l'Assemblée suprême sanitaire (Sobre los avatares de la junta suprema sanitaria), Voir RodRíGuEz OCAÑA, Esteban, "El resguardo de la salud. Organización sanitaria española en el siglo XVIII ", Dynamis, 7-8, 1987-1988, p. 145-170, p. 150 sq. ; y BETRán MoYA, José Luís, La peste..., op. cit., p. 229-232.

105. Sa création obéit à un ordre royal du 30 septembre 1800 , par lequel on a déterminé que face au danger d'apparition de la fièvre jaune, on généralisera la création de ces assemblées dans les capitales des provinces et les villages têtes de partis. RoDRíGUEZ OCAÑA, Esteban, Ibidem, p. 150. Par rapport à celle de Valladolid, qui fut l'une des premières créées, celle de León présente un retard de presque une décennie. MERCHÁN FERNANDEZ, A. Carlos, Gobierno Municipal..., op. cit., p. 286. 
maient qu'ils avaient été mis au courant ${ }^{106}$. En outre, L’Assemblée, assistée par les médecins et les chirurgiens de la ville, signalait les maisons où devaient être mis en quarantaine les malades et les suspects de contagion, bien entendu, loin des noyaux habités, la manière de les déplacer jusque-là, etc. Vu que la ville de Léon était connue pour être malsaine et que, sans aucun doute, elle dépassait régulièrement beaucoup d'autres agglomérations en nombre et en durée des maladies, le 10 février 1801, un rapport a été élaboré, où sont mentionnées les maladies qui y sont les plus fréquentes et les mois pendant lesquels elles s'attaquaient aux habitants.

Les bulletins sont déjà le fruit du nouveau régime libéral. Dans celui du 27 mai 1834 sont repris les conseils et les mesures d'hygiène qui prétendent trouver un écho dans toute la province dans le but d'éviter et de traiter les fièvres paludiques. On y parle de propreté des rues, d'entretien des sources et des lavoirs, de drainage des eaux stagnantes, d'arrosage des prés, d'enterrement des bêtes mortes, etc. Dans celui du 8 juillet 1834, on traite des cordons sanitaires pour éviter que le choléra morbus n'infecte la province. Dans certains cas, comme celui du 15 juillet de cette même année, les avertissements vont au-delà de tout cela. On y fait des recommandations sur l'hygiène domestique ou alimentaire. Les édits municipaux vont dans la même direction que les bulletins provinciaux.

Avec l'avènement du XIX siècle, les progrès dans le domaine de la médecine furent nombreux, mais le peuple les accueillit avec une certaine méfiance ${ }^{107}$. Le vaccin contre la variole, découvert à la fin du siècle précédent, aurait pu éviter de grandes mortalités infantiles. En 1804, Charles IV ordonna que cette immunisation soit appliquée, gratuitement, dans les hôpitaux des capitales des provinces, mais il paraît que la mesure n'a pas eu l'accueil espéré. Cette même année, le conseil municipal de Léon insista sur les effets bénéfiques du vaccin ${ }^{108}$ antivariolique. En 1805, on demanda aux curés ${ }^{109}$ d'insister auprès des paroissiens, pendant les homélies, pour qu'ils vaccinent leurs enfants et, en 1834, on renouvela ce conseil ${ }^{110}$. Malgré les bonnes intentions des dirigeants de Léon, les résultats de toute cette législation furent décourageants.

106. AHPL, C. 4886.

107. Voir ToRRES SÁnCHEZ, Rafael, Ciudad y población. El desarrollo demográfico en Cartagena durante la Edad Moderna, Ayuntamiento de Cartagena, Cartagène, 1998, p. 135.

108. AHML, C. 82.

109. ADL, Libros Parroquiales de Santa María del Mercado.

110. FernándeZ ARIENZA, José, Medicina y sociedad..., op. cit., p. 21 sq. 


\section{RÉSUMÉ}

Durant l'époque moderne, la ville de Léon a souffert plusieurs épisodes de mortalité catastrophiques, aggravés par l'arrivée d'un grand nombre de pauvres affamés provenant des montagnes de Léon et de la Principauté des Asturies. Léon, comme d'autres villes de l'époque, n'avait pas d'infrastructures institutionnelles pour absorber cette masse humaine. Mais les indices de mortalité élevés ne sont pas liés seulement aux processus épidémiologiques car, même dans des conditions normales, la croissance naturelle de la population pouvait être affectée par l'insalubrité générale dans laquelle se déroulait le cycle vital, en raison du manque d'hygiène publique et privée et de l'absence d'une infrastructure urbaine adéquate.

\section{ABSTRACT}

During the Modern Age, the city of León suffered from several episodes of catastrophic mortality. These were made worse by the arrival of a great number of poor and starving people who came from the mountains and the Principado de Asturias. As many other cities at that time, Léon lacked institutional infrastructure to soak up that human mass. However, the high death rates weren't only related to epidemiologic processes; even in normal conditions, the vegetative growth used to be affected by the generalized insalubrity which life took place in, owing to the lack of public and private health control as well as the need for an appropriate urban infrastructure. 